\title{
IVORY EXOSTOSIS OF THE SKULL
}

\author{
Peter G. Bullough, M.B., Ch.B., \\ The Nuffield Department of Orthopadic Surgery, University of Oxford.
}

THE IVORY exostosis, or, as it is sometimes called, the ivory osteoma, is a bump of dense bone most often seen on the cranial vault (Fig. 1) or in relation to one or other of the nasal sinuses (Fig. 2). In keeping with its usual origin from a bone preformed in membrane it is not capped by cartilage as is the osteochondroma or exostosis which is commonly seen arising from a long bone.

In hospital practice ivory exostoses are very rare, though probably there are many cases which are non-symptomatic. Smith (1952), reporting from Duke University found a total of forty cases in 176,000 surgical specimens examined in their pathology department over a twenty year period.

The purpose of this paper is to discuss the clinical presentation and the pathology of the exostoses of twenty-one patients treated surgically over the last twelve years in Oxford. In seven the tumour arose from the cranial vault and in fourteen was related to a nasal sinus. In the first group there were no symptoms and usually the lesions were removed for cosmetic effect. In the second group the patients complained of stabbing frontal headaches, bulging of the eye (Fig. 3), recurrent infection of the sinuses and alteration in vision. The left frontal sinus was affected much more frequently (ten cases) than the other nasal sinuses.

Møst of the patients were between twenty and forty years of age; the youngest was twelve years and the oldest 68 years. In the first group presenting with cranial lesions six were female, whereas in those presenting with lesions of a sinus ten were male.

The following selected cases illustrate the salient features of these two types of lesion.

\section{Case Reports}

\section{Case No. 1}

An eighteen-year-old white female who worked as a shorthand typist. About two years prior to admission her hairdresser had noticed a lump on the right side of her head. Since then she had "worried" about it and she thought that possibly it was growing larger. There were no other symptoms.

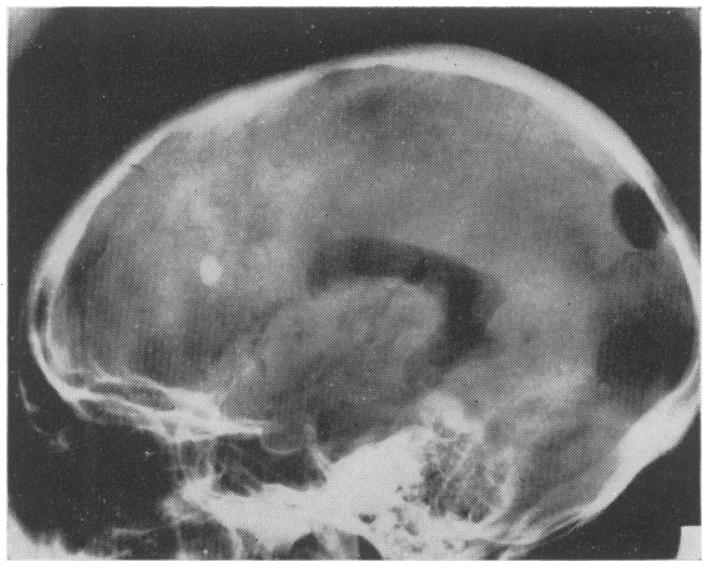

FIG. 1.-X-ray of an exostosis of the parietal bone in a forty-year-old woman.

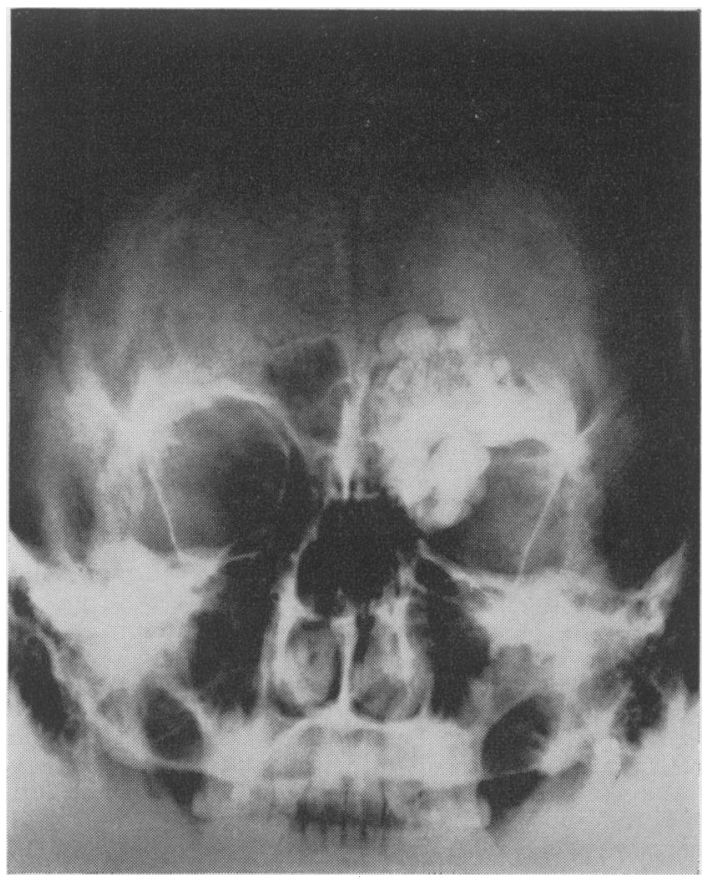

FIG. 2.-X-ray of an exostosis of the left frontal sinus in a twenty-nine-year-old man. 


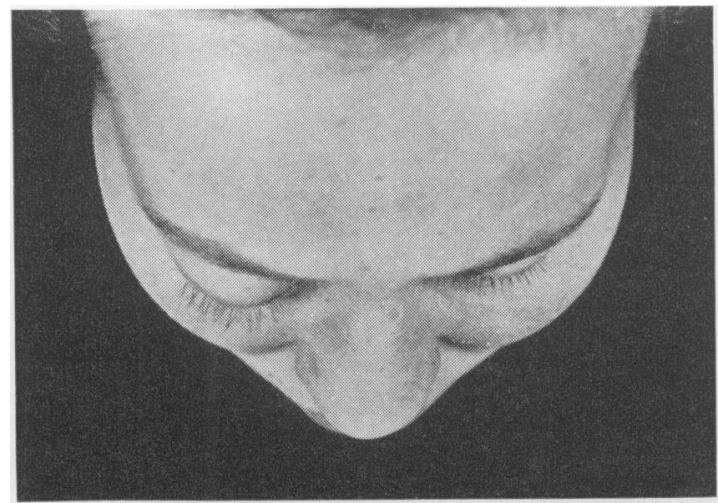

Fig. 3.-Clinical photograph of a twenty-six-year-old woman complaining of recurrent rhinitis and diplopia. A large exostosis was removed from the right frontal sinus.

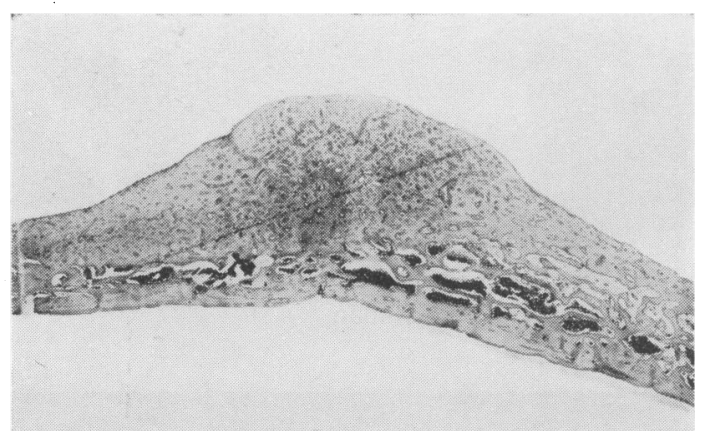

FIG. 4.-Macro-photograph of an exostosis removed from the right parietal bone of an eighteen-yearold girl. (H. \& E. X4).

On examination there was slight tenderness over the bump, the skin moved freely over it and X-ray examination revealed an ivory osteoma of the parietal bone. Histologic examination revealed dense cortical bone but no other abnormality (Fig. 4).

\section{Case No. 2}

A seventeen-year-old white male working as a farm labourer. Five years prior to admission he first noticed bulging of the left eye and at about the same time he started to experience stabbing pains over the left eye. His local doctor diagnosed the condition as migraine and treated him with phenobarbitone. After six months the pains ceased and did not return until two months prior to admission. He had never had fits.

On examination the left eye was protuberant by five millimetres. All the movements of the left eye were slightly limited and there was diplopia at the extremes of gaze in all directions. An X-ray picture of the skull showed a radio-opaque lesion of the left ethmoid sinus encroaching on the left orbit. At operation the tumour had eroded the orbital plate and the outer layer of the dura mater so that the arachnoid was in contact with the tumour. The histology of the lesion is illustrated in Figs. 6-10.

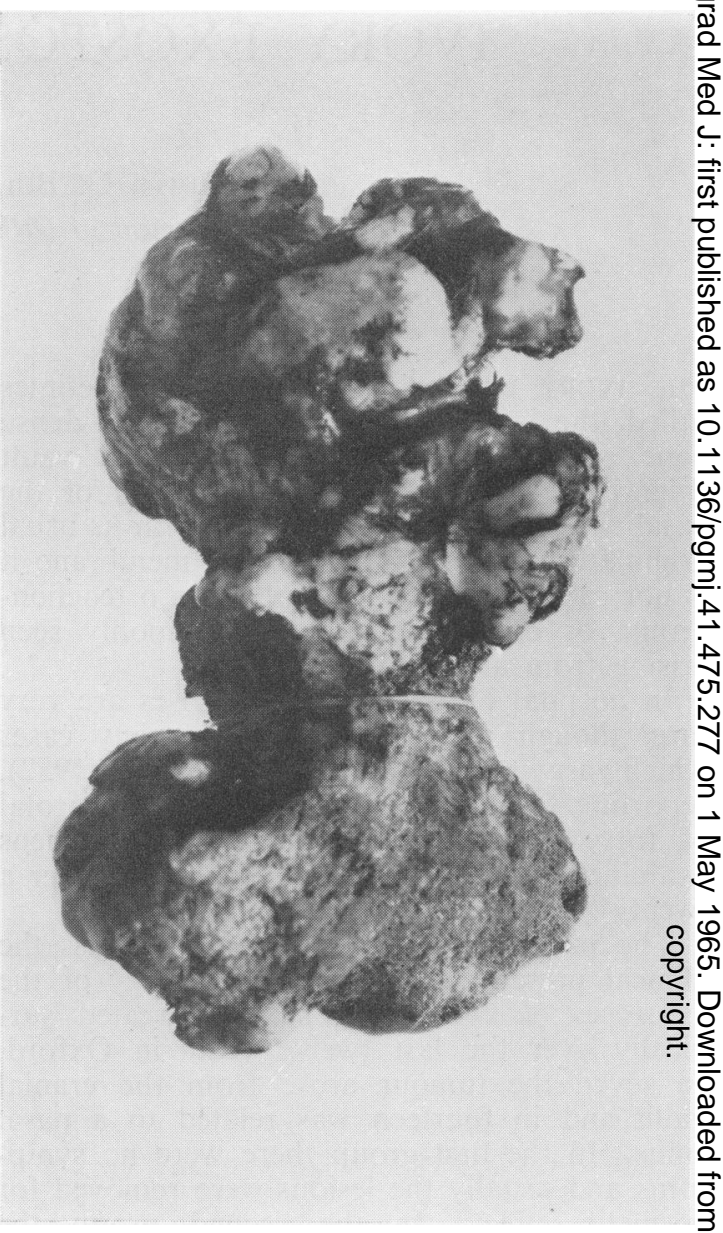

Fig. 5.-Photograph of the tumour removed from? the left frontal sinus of a fifty-year-old man witlo़ recurrent sinusitis.

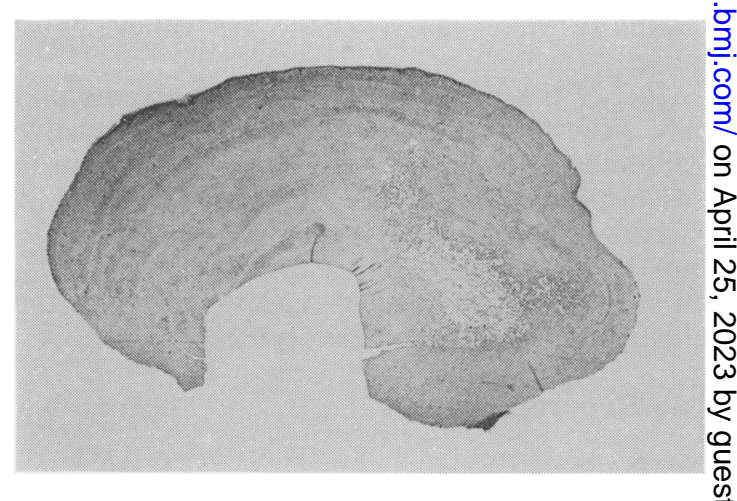

FIG. 6.-Macrophotograph of an exostosis removed from the left ethmoid sinus of a seventeen-year $-{ }^{\circ}$ old boy complaining of headaches and bulgingo of the left eye for approximately five years. (H. \& E. x4). 


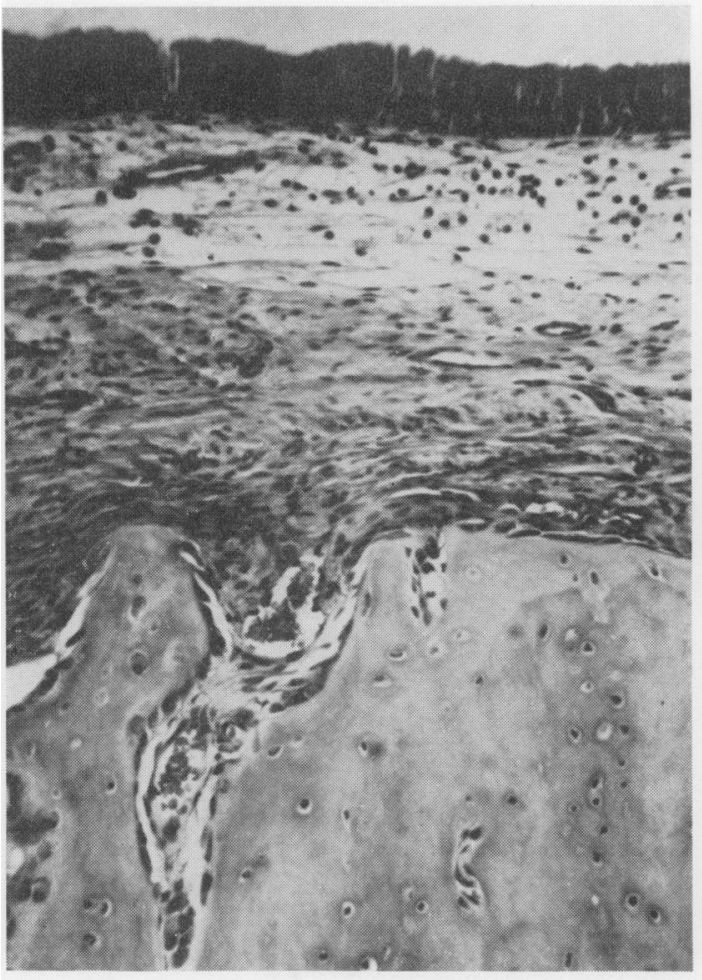

(a)

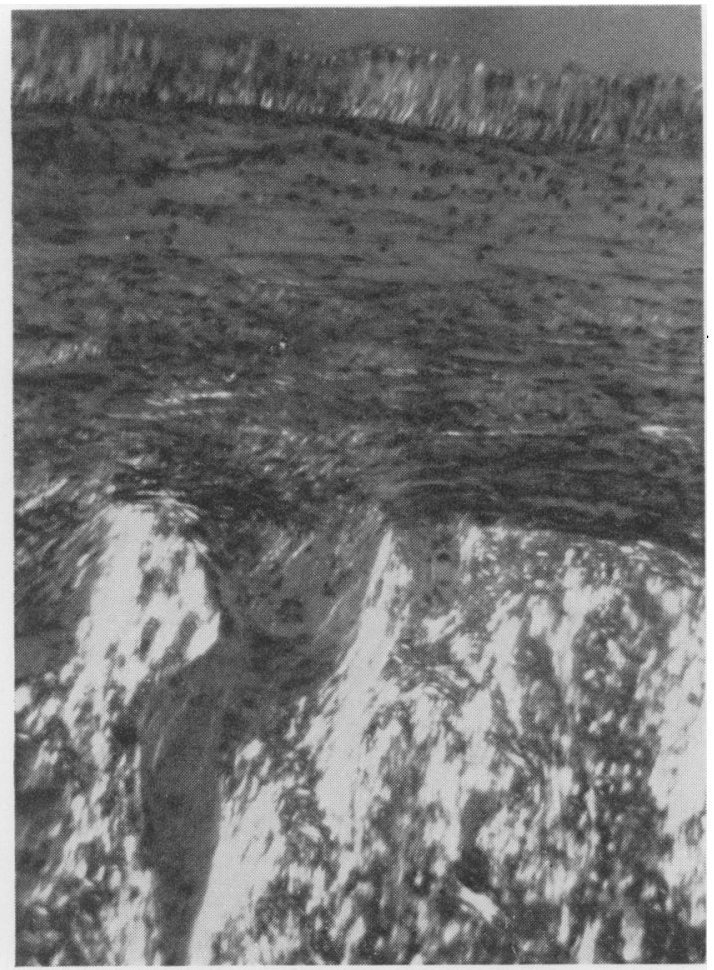

(b)

Figs. 7a \& b.-Photomicrograph of the edge of the lesion shown in Fig. 5. There is a very mild chronic inflammatory infiltrate beneath the respiratory epithelium lining the sinus. Fig. 7b taken of the same field by polarized light shows the non-lamellar patttern of the collagen in the bone matrix. (H. \& E. X400).

Case No. 3

A thirty-five-year-old white farmer. Two months prior to admission he noticed that in order to keep the sun out of his eyes it had become necessary to tip his hat more and more. At the same time he had noticed some slight pain around the eye. At the eye hospital he was told that there was a slight displacement of the eye. Study of photographs taken two years before the onset of symptoms showed in retrospect that even then there had been some displacement.

On examination it was found that there was a hard, non-tender, bony lump in the supero-medial part of the right orbit. The proptometer reading was fifteen millimetres on the right and thirteen millimetres on the left. The X-ray picture showed a lobulated radio-opaque mass filling the right frontal sinus. At operation the lesion had eroded through the dura mater. Histologic examination revealed an essentially similar picture to the previous case except that the laying down of lamellar bone in the central part of the lesion had proceeded somewhat further. He was admitted to hospital for a second time six months later with almost complete ptosis of the right eyelid and pain over the eye. An X-ray picture revealed that a good deal of the lesion still remained in the sinus and he was re-explored and the rest of the lesion removed.

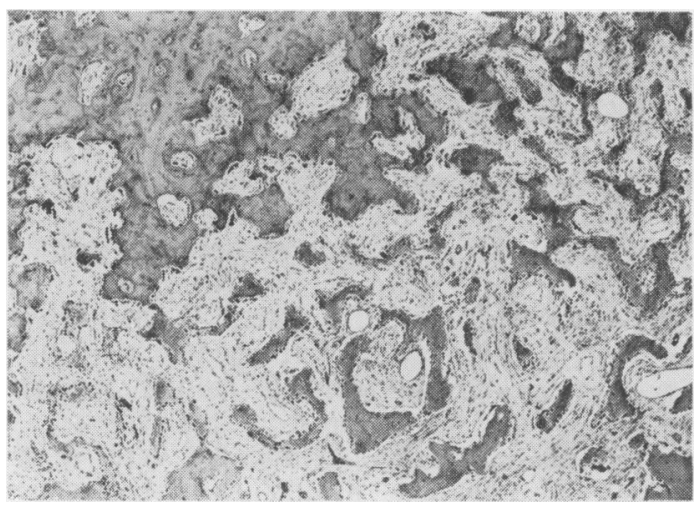

FIG. 8.-Photomicrograph of the central area of the lesion shown in Fig. 6. There is active osteoclastic resorption of the non-lamellar bone in the upper right hand corner of the picture and replacement by vascular fibrous tissue in which lamellar bone trabeculæ are being formed. (H. \& E. x100). 


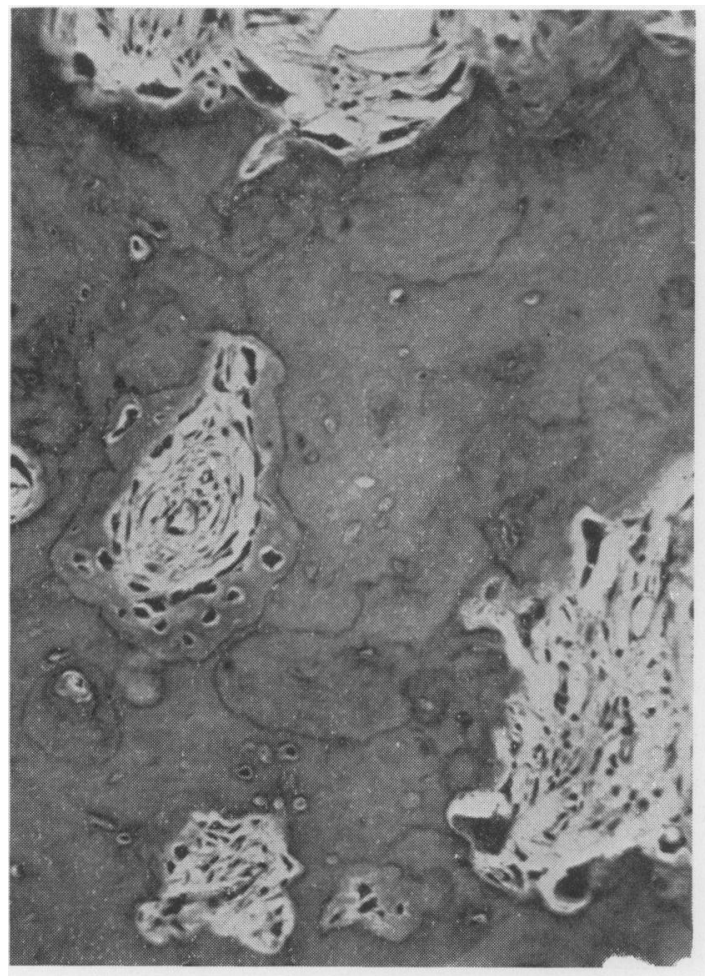

(a)

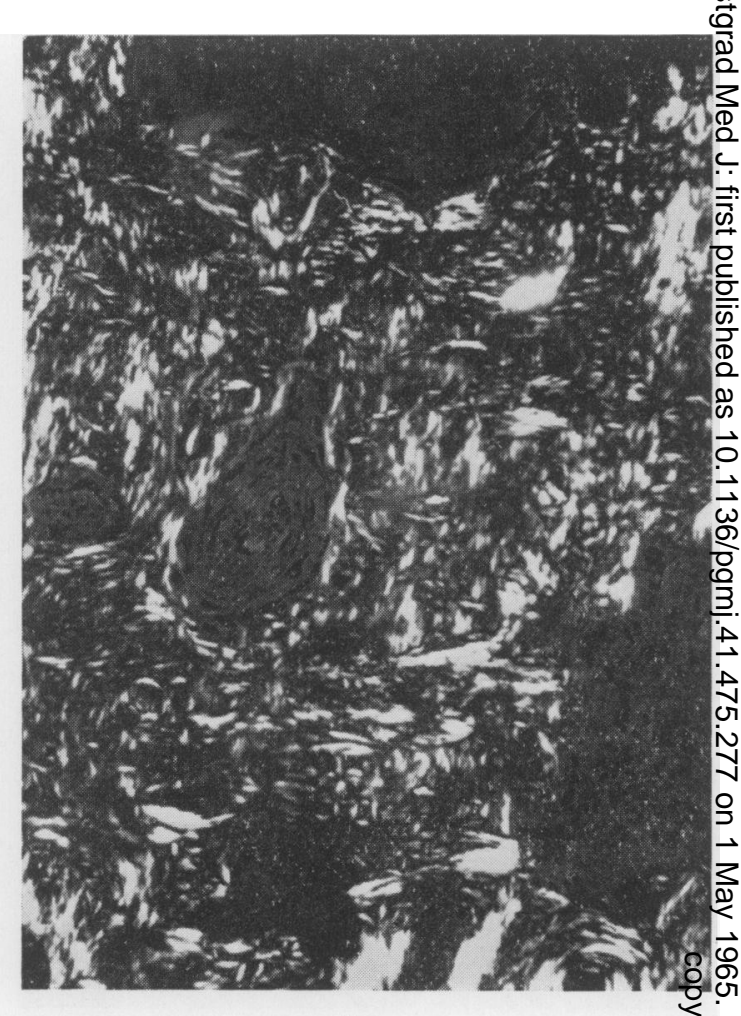

(b)

FIGS. 9a \& b.-Photomicrograph at higher power of the right hand corner of Fig. 8 showing the osteoclastic resorption and in $9 \mathrm{~b}$ taken by polarized light to demonstrate the nonlamellar character of the bone. (H. \& E. X400).

\section{Discussion}

In this series the two principal complications of the tumours occurring in the nasal sinuses were erosion of the bone, particularly the supraorbital plate, and subsequent erosion of the dura mater, and secondly, blockage of the nasal duct. The first sometimes resulted in severe bleeding and difficulties in approach at the time of operation, the second in recurrent attacks of sinusitis and sometimes the formation of a mucocele. Hudolin, Riessner, Kadrnka and Knezevicm (1961) have reported a case of a large osteoma arising from the frontal sinus and extending into the anterior cranial fossa in a patient who presented clinically with severe mental derangement, headaches, incontinence, epilepsy and chronic alcoholism. She was considerably improved after the removal of the lesion.

The differences in clinical presentation depending upon the site of the lesion are to be expected, but morphologically there are also marked dissimilarities.
The lesions which occur on the vault of the skull are generally smaller, usually about one centimetre in diameter, and are smooth and regular in outline (Fig. 4). They are formedo of non-lamellar woven bone. When examined. in situ they are poorly vascularized and appears very pale against the surrounding skull.

The tumours occurring in relation to the sinuses (Fig. 5) are larger, irregular in outline and hyperemic. They consist largely of primi-o tive woven bone (Fig. 6). At the edge of the lesion there is evidence of active production: of woven bone which is recognised histologically, by the large closely packed osteocytes and the irregular pattern of the collagen (Figs. 7a ando $7 b$ ). Away from the periphery of the tumour most of the osteocytes are dead. In the centraR area there is active resorption of the bone witke the subsequent deposition of adult lamellaro bone (Figs. 8, 9a and 9b, 10).

Taken as an isolated histologic field Fig. $8^{\circ}$ has the appearances of Paget's disease in the active phase and indeed at the periphery of the $\frac{\mathrm{O}}{\mathrm{O}}$ 


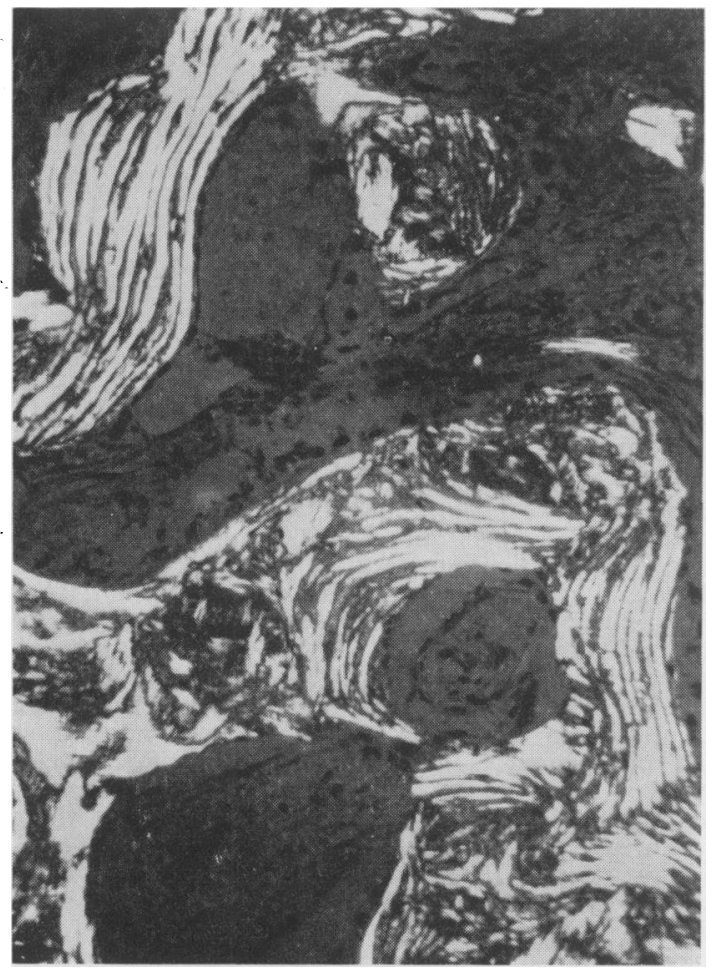

FIG. 10.-Photomicrograph at higher power of the trabeculæ being laid down in the vascular fibrous tissue illustrated in Fig. 8 to show the lamellar character of the bone being formed. (H. \& E. $\mathrm{x} 400$ ).

hypervascular fibrous zone there is even a mosaic appearance. It is because of this appearance that the lesion has been interpreted as fibrous dysplasia, localised leontiasis ossea 'and Pagetoid bone. But in fact the histologic appearances are consistent with what we know of the remodelling and replacement of woven bone by lamellar bone in other situations, for example, myositis ossificans circumscripta, or fracture callus (Collins and Curran, 1959).

The hyperactive periosteum lays down a bump of woven or non-lamellar bone which acts as a calcified scaffold upon which the vascularized fibrous tissue can build the lamellar bone. The lesion therefore follows a general rule that lamellar bone is only laid down on a pre-existing calcified framework, either calcified cartilage, calcified woven bone, or foci of dystrophic calcification.

In the ivory exostosis of the cranial vault the growth is probably very slow and the substitution of the woven bone by lamellar bone occurs at a steady pace. Thus these lesions show mostly a lamellar pattern with only occasional fragments of non-lamellar bone. On the other hand the lesions occurring in relation to the sinuses have a more rapid growth and depending on the speed of growth and the age of the lesion will show different proportions of the two types of bone. Because of their position the lesions of the sinuses will also tend to draw attention to themselves much sooner than the calvaral lesions.

Both types of the tumour probably have their origin in stimulation of the periosteum which lays down a non-lamellar type of bone. The stimulation is probably due to a developmental abnormality, trauma or possibly infection. Maxwell (1946) has suggested that the tumours found in relation to the paranasal sinuses are the result of a chronic proliferative osteomyelitis secondary to sinus infection. In the cases reported here, however, infection did not seem to be a predisposing factor and this was also the experience of Andrew (1956).

\section{Summary}

1. Twenty-one cases of exostosis of the skull are presented. Fourteen cases were found in relation to one or other of the paranasal sinuses, most frequently the left frontal sinus, and seven were on the cranial vault.

2. Depending upon the site the clinical presentation of the tumour differs.

3. The pathological appearance of the lesions also differs, both on gross and microscopic examination.

4. Despite the superficial dissimilarities evidence is presented to support the view that the exostosis of the cranial vault and of the paranasal sinuses are basically similar.

\section{REFERENCES}

ANDRew, J. (1956): Osteomata of the Paranasal Sinuses, Brit. J. Surg., 43, 489.

Collins, D. H., and CURRAN, R. C. (1959): Pathological Ossification and Osseous Metaplasia in Man in 'Modern Trends in Pathology'; Chapter 15; London: Butterworth.

Hudolin, V., RIESSNER, D., KADRNKA, S., and KNEZEVICM (1961): Osteoma in the Anterior Cranial Fossa, J. Neurol. Neurosurg. Psychiat., 24, 80.

Maxwell, J. H. (1946): Chronic Proliferative Osteomyelitis of the Skull, Ann. Otol. (St. Louis), 55, 719.

Smith, A. G., and Zavaleta, A. (1952): Osteoma, Ossifying Fibroma, and Fibrous Dysplasia of Facial and Cranial Bones, A.M.A. Arch. Path., 54, 507. 